\title{
ADHD Symptoms and School Impairment History in Parents of ADHD Children are a Fundamental Diagnostic and Therapeutic Clue.
}

\author{
Luisa Cortellazzo Wiel ( $\sim$ luisacortellazzowiel@gmail.com ) \\ University of Trieste https://orcid.org/0000-0003-0069-560X
}

Francesco Rispoli

University of Trieste Department of Surgical Medical and Health Sciences: Universita degli Studi di

Trieste Dipartimento Universitario Clinico di Scienze Mediche e Chirurgiche e della Salute

\section{Giulia Peccolo}

University of Trieste Department of Surgical Medical and Health Sciences: Universita degli Studi di

Trieste Dipartimento Universitario Clinico di Scienze Mediche e Chirurgiche e della Salute

\section{Valentina Rosolen}

Institute for Maternal and Child Health: IRCCS materno infantile Burlo Garofolo

\section{Egidio Barbi}

University of Trieste Department of Surgical Medical and Health Sciences: Universita degli Studi di Trieste Dipartimento Universitario Clinico di Scienze Mediche e Chirurgiche e della Salute

\section{Aldo Skabar}

Institute for Maternal and Child Health: IRCCS materno infantile Burlo Garofolo

\section{Research}

Keywords: Attention Deficit Hyperactivity Disorder, Familiarity, Wender Utah Rating Scale-25, School impairment, Emotional lability

Posted Date: November 25th, 2020

DOl: https://doi.org/10.21203/rs.3.rs-113397/v1

License: (c) (i) This work is licensed under a Creative Commons Attribution 4.0 International License. Read Full License

Version of Record: A version of this preprint was published at Italian Journal of Pediatrics on March 28th, 2022. See the published version at https://doi.org/10.1186/s13052-022-01240-7. 
TITLE PAGE

Title: ADHD symptoms and school impairment history in parents of ADHD children are a fundamental diagnostic and therapeutic clue.

\section{Authors:}

Luisa Cortellazzo Wiel, MD¹, luisacortellazzowiel@gmail.com

Francesco Rispoli, MD ${ }^{1,}$ francesco.rispoli@outlook.it

Giulia Peccolo, MD¹, giupeccolo@gmail.com

Valentina Rosolen, $\mathrm{MD}^{2}$, valentina.rosolen@burlo.trieste.it

Egidio Barbi, Prof, MD ${ }^{1,2}$, egidio.barbi@burlo.trieste.it

Aldo Skabar, MD², aldo.skabar@burlo.trieste.it

\section{Affiliations:}

1. University of Trieste, Trieste, Italy

2. Institute for Maternal and Child Health - IRCCS "Burlo Garofolo", Trieste, Italy

Corresponding author: Luisa Cortellazzo Wiel, MD, University of Trieste, Piazzale Europa 1, 34127 Trieste, Italy 


\section{ABSTRACT}

Background: Attention Deficit and Hyperactivity Disorder (ADHD) is a multi-factorial condition, with inheritance playing a major role. Recognizing parents' ADHD represents a clue not only for an earlier diagnosis of the disease in their children, but also to optimize psycho-educational therapy outcome, by addressing the impairment of parenting related to untreated ADHD. This study aimed to assess the frequency of features suggestive of ADHD during childhood among parents of affected children, and the presence of school and emotional impairment.

Methods: We administered the Wender Utah Rating Scale-25, a self-assessment tool for the retrospective identification of symptoms consistent with ADHD during childhood, to a cohort of 120 parents of 60 children diagnosed with ADHD, and to a consistent number of "controls".

Results: The WURS-25 proved positive in $49.1 \%$ of fathers and $30.0 \%$ of mothers of ADHD patients, compared to $1.7 \%$ of fathers and $1.7 \%$ of mothers of non-ADHD patients $(p<0.0001)$.

The questions addressing learning and emotional impairment provided significantly higher scores in parents with an overall positive test compared to those with negative test $(p<0.0001)$.

Conclusions: This study demonstrates a remarkably high rate of symptoms consistent with ADHD during childhood in parents of affected children. Physicians should be aware that this is a relevant anamnestic clue and, given the relevance of parents' role in the management of children with ADHD, an important issue in order to improve patients' treatment.

Keywords: Attention Deficit Hyperactivity Disorder; Familiarity; Wender Utah Rating Scale-25; School impairment; Emotional lability 
TEXT

\section{Background}

Attention Deficit and Hyperactivity Disorder (ADHD) is a condition characterized by marked, persistent, maladaptive levels of inattention, impulsiveness, and hyperactivity, which has a negative impact on social, educational, and professional performances. Its estimated prevalence worldwide is $5 \%$ in children and $2.5 \%$ in adults ${ }^{1}$, with studies showing that in half the cases, the disorder persists during adulthood ${ }^{2,3}$. According to a survey ${ }^{4}, 40 \%$ of children diagnosed with ADHD undergo remission during adulthood, in $40 \%$ of cases symptoms persist in an attenuated form with related emotional deregulation, social and professional difficulties, while $20 \%$ continue to show features consistent with the typical form of the disease.

Although not yet fully understood, ADHD aetiology is probably multi-factorial. Several factors have been demonstrated to be involved, including low birth weight, smoking, drug and alcohol exposure during pregnancy, among biological factors ${ }^{5}$ and maternal psychiatric disorders, family dysfunction and lower socioeconomic status among environmental modifiers ${ }^{6}$. The identification of specific causative genes is still hampered by the significant phenotypic heterogeneity of the disorder ${ }^{7}$. Nevertheless, the role of inheritance has been well clarified ${ }^{8}$ : twin concordance amounts to $70-76 \%^{9,10,11}$ and the presence of an affected first-degree relative has been demonstrated to give four times higher risk, to any child, to develop the disorder ${ }^{12}$.

This study aimed to assess the frequency of features suggestive of ADHD during childhood among parents of affected children, and the presence of school difficulties and emotional lability.

\section{Methods}

A prospective case-control study was performed at the Child Neurology and Psychiatry Unit of the University teaching, tertiary children's hospital, Institute for Maternal and Child Health "Burlo Garofolo", in Trieste, Italy, from April 2019 to October 2019. The study was approved by the Institutional Review Board and all participants gave their written consent to take part in it. 
We considered as "cases" the parents of children, who had received a diagnosis of ADHD (of any of the following types: impulsive/hyperactive, inattentive and distractible, combined) between January 2005 and June 2019. The control group consisted of parents of children with various neuropsychiatric conditions, summarized in Figure 1, and did not include those of patients who presented other conditions leading to attention or behavioural deficits, potentially acting as confounding factors. Furthermore, since all ADHD patients had an intellective quotient (IQ) over 70, we selected comparable controls, thus excluding also parents of patients with $I Q \leq 70$.

We initially identified 202 patients; 142 were not included due to the presence of exclusion criteria or to the impossibility to contact their families. Sixty ADHD children (56 males) were therefore finally considered, along with 120 parents (60 mothers and 60 fathers): the latter were considered as "cases" and were matched to 60 mothers and 60 fathers of non-ADHD children as a control group.

Regarding the disease subtypes, $2 / 60$ (3.3 \%) children had predominant hyperactivity, 14/60 (23.3\%) predominant inattention, and $44 / 60(74.3 \%)$ a combined disorder. The $44 / 60(73 \%)$ of children had at least one co-morbidity: 35/60 (58\%) of patients displayed Oppositional Defiant Disorder, 11/60 (23\%) Specific Learning Disorder, 10/60 (17\%) Mood or Anxiety Disorder, and 7/60 (12\%) other disorders, such as mixed learning disorder, language disorder, autism, epilepsy or obsessive-compulsive disorder, and 17/60 (28\%) of enrolled patients had more than one co-morbidity. Finally, 46/60 (77\%) of children with ADHD were on ongoing drug therapy with methylphenidate, in 15/60 (25\%) in association with an antipsychotic drug, with one patient taking risperidone as a single drug; $13 / 60$ (22\%) were not receiving any drug therapy.

The Wender Utah Rating Scale-25 (WURS-25) $)^{13,14}$, in the Italian language (Figure 2), was administered online to all participating parents. The WURS-25 is a self-assessment tool for the retrospective identification of the presence and severity of symptoms consistent with ADHD during childhood (age 6-10 years). It consists of 25 items, of which 21 address ADHD (inattention, hyperactivity, impulsivity, affective, emotional, and functional dysfunction), and four serve as control items. This tool has shown good psychometric properties and satisfactory internal and temporal reliability, and it is considered a screening tool for the retrospective assessment of ADHD in childhood. It has been demonstrated that ADHD symptoms undergo remission through adulthood in a relevant percentage of the cases. Therefore, when 
assessing the presence of symptoms suggestive of ADHD in the adult population, there is a substantial risk of underestimating the real prevalence of the disorder in childhood, making WURS a more suitable tool for this study, providing an accurate picture of the period in which the disorder may have been more expressed.

We instructed the subjects to recall their behaviour and mood during primary school (age 6 to 10), rating every item from 0 to 4 (not at all or very slightly (score=0), mildly (score=1), moderately (score=2), quite a bit (score=3), or very much (score=4)). The total score range from 0 to 100: we considered 46 as the cut-off score suggestive for previous ADHD.

Four questions (number 22-25) aimed to evaluate scholastic impairment, while seven questions (number 2$3,9,11-13,17)$ concerned the presence of emotional lability.

We used the Chi-Square Test for the dichotomous variables and the Fisher exact test in case of frequencies below 5. For continuous variables, we used the Wilcoxon-Mann Whitney test (for all distributions, the Kolmogorov-Smirnov test for normality had a $\mathrm{p}$-value $<0.05)$.

Results with $p$-value $\leq 0.05$ were considered statistically significant.

\section{Results}

Among parents of ADHD children, $60 / 60$ mothers (100\%) and $57 / 60$ fathers (95\%) answered the questionnaire, versus 60/60 (100\%) and 60/60 (100\%) mothers and fathers of non-ADHD children, respectively.

The test proved positive (score $\geq 46$ ) in $46 / 117$ parents of children with ADHD (39.3\%) and 2/120 (1.7\%) parents of non-ADHD children $\left(\chi^{2}=51.99, p<0.0001\right)$ (Figure 3$)$.

Among parents of ADHD children, we detected a higher rate of positivity in fathers $(28 / 57,49.1 \%)$ compared to mothers $(18 / 60,30.0 \%)\left(\chi^{2}=4.48, p=0.034\right)$, while this difference was not observed among controls $\left(1 / 60,1.7 \%\right.$ fathers and $1 / 60,1.7 \%$ mothers; $\left.\chi^{2}=0.000, p=1.000\right)$. 
As for mothers, $94.7 \%$ of the positive tests belonged to "the case group", which meant that they had a child with $\operatorname{ADHD}\left(\chi^{2}=17.01, p<0.0001\right)$. Similar results were observed in fathers, with a slightly higher correlation $\left(96.6 \%\right.$ vs. $\left.3.4 \%, \chi^{2}=29.43, p<0.0001\right)$.

In parents tested positive, a concomitant compromise of the school and emotional functioning was sought. The questions, aimed at investigating school impairment, provide a score ranging from 0 to 16 points. The median score was 8 (IQR 7-10) among the 48 parents with overall WURS positive test, versus 3 (IQR 1-4) among the 189 parents testing negative (Table 1, Figure 3). Of interest, $28.3 \%$ of parents with overall WURS positive test, repeated at least one class. Through the Wilcoxon-Mann-Whitney test, we compared the scores regarding school impairment of parents with an overall positive and negative WURS test, finding significantly higher scores among parents with a positive test $(p<0.0001)$. The median score was 9 (IQR 712) among the 19 mothers who tested positive, against 3 (IQR 1-4) among the 101, who were negative. Statistically a significant difference was found between the two groups $(p<0.0001)$. Besides, $26.3 \%$ of mothers with positive tests reported having repeated at least one class during the school period. The median score of fathers was 8 (IQR 7-9) among the 29 who tested positive, versus 3 (IQR 2-5) among the 88 who tested negative. Even in this case, there was a statistically significant difference between the two groups ( $p<0.0001$ ), and again, the $27.6 \%$ of fathers with positive tests reported having repeated at least one class during the school's years.

The questions related to the presence of emotional lability, provide a total score between 0 and 28 points. The median score was 14 (IQR 11.5-15) among the 48 parents with positive tests, versus 5 (IQR 3-7) among the 189 , who were negative. The difference was statistically significant $(p<0.0001)$ (Table 2, Figure 3$)$. The median score of mothers was 14 (IQR 14-15) among the 19 who were positive, versus 5 (IQR 3-7) among the 101 , who tested negative $(p<0.0001)$. For fathers, the median score was 13 (IQR 10-15) among the 29 who were positive, versus 5 (IQR 3-7) among the 88 , who proved to be negative $(p<0.0001)$. 


\section{Discussion}

This study shows a high rate of symptoms consistent with ADHD during childhood in parents of children affected by this disease.

Several aspects are involved in the multi-factorial pathogenesis of ADHD, including genetic, neurobiological and environmental elements. Inheritance probably plays a fundamental role in pathogenesis: the assessment of its weight would allow not only a better understanding of the disease but also an early diagnosis in the children of affected parents, along with timely treatment and prevention of detrimental consequences. Remarkably, a positive family history may be a relevant diagnostic clue, which physicians should specifically address. On the other hand, parent training is a cornerstone of treatment for children with mild-to-moderate $A D H D^{15}$. Since parental $A D H D$ may particularly impair parenting and family functioning, hindering the ability to deal with affected children, identifying affected parents could be a clue to optimize patients' outcomes ${ }^{16,17}$.

In this study we found a significant correlation between kinship and parents' WURS test positivity: in particular, $49.1 \%$ of fathers and $30.0 \%$ of mothers of ADHD patients, compared to $1.7 \%$ of fathers and $1.7 \%$ of mothers of non-ADHD patients, had a positive test, presenting features consistent with ADHD during their childhood. Remarkably none of the analyzed parents had ever been diagnosed with ADHD, underlying a lack of standardized diagnostic criteria to detect this condition in the past. However, the WURS test does not allow the retrospective diagnosis of ADHD: indeed, its functionality is to highlight the presence of emotional and behavioural traits consistent with the disease. These results are in line with the previous literature. The role of genetic factors has been assessed over time ${ }^{8}$, and it has been confirmed by the high concordance between twins ${ }^{3,9,11}$. Bidwell demonstrated a four-time higher risk of developing ADHD in children with affected parents or first-degree relatives, compared to the general population ${ }^{12}$. Starck and colleagues found a WURS test positivity in $49.1 \%$ of fathers and $27.3 \%$ of mothers of ADHD patients ${ }^{18}$. Compared to the survey mentioned above, our study was powered by the analysis of data from a control group and by the comparable number of enrolled fathers and mothers, which allowed us to stratify the 
weight of familiarity by the sex of affected parents. While previous studies relied on the WURS test to assess symptoms suggestive of ADHD during childhood in parents of affected children, to our knowledge, this is the first study that separately examines the school and emotional impairment of parents during their childhood, through a specific sub-analysis of the scores obtained in the specific test questions, addressing these issues.

Regarding school performances, we found a correlation between reported parents' school difficulties and WURS test positivity, confirming the pivotal role of undiagnosed and untreated ADHD in learning problems. The latter, as underlined by Marzocchi and colleagues ${ }^{7}$, can be explained in the light of a vicious circle between the deficit of self-regulation cognitive processes and the inability to adopt effective organizational and executive strategies appropriate to the task. This profile negatively affects the performance in the comprehension of written texts, studying, and resolution of arithmetic problems ${ }^{19,20}$. In this study, we found that mothers with positive tests showed higher scores in the questions investigating school impairment, compared to fathers, whose tests were positive. This issue could further demonstrate that in females with ADHD, inattention is usually preponderant compared to hyperactivity, negatively affecting academic performances. Similar results were found regarding emotional deregulation. Parents with an overall positive WURS test demonstrated higher scores in the questions related to emotional lability, compared to parents with negative tests.

Tabassam and Grainger widely described the emotional dysfunction of ADHD children, characterized by sudden emotional changes, dysphoria, irritability, low tolerance to frustration, emotional hyper-reactivity, and emotions-recognition deficits ${ }^{21}$. The concurrent effect of the critical judgment of families, teachers, and peers, easily led to low self-esteem, sense of social rejection and loneliness, which in turn can promote the development of further psychopathologies, such as mood disorders. By stratifying data by gender, we found that the mothers tested positive presented a median score higher compared to fathers tested positive, underlying the central role of emotional lability within the disorder in females. In a longitudinal study involving 140 children diagnosed with ADHD, Hinshaw found that female subjects had more severe anxiety, depressive symptoms, and more significant difficulties in daily functioning than males ${ }^{22}$. At ten 
years of follow-up, girls with ADHD were more likely to exhibit self-injuring behaviors and suicide attempts than boys.

This study, not only confirms these data but also highlights a high occurrence of school and emotional impairment in parents of affected children during their childhood, strengthening the suitability of the WURS test for the retrospective assessment of symptoms suggestive of ADHD, in light of the crucial role of these two aspects in the diagnosis of the disorder.

The main limits of this study consist of the relatively small sample size and the lack of correlation analysis between patients and parents, due to the choice to collect and investigate the results in a completely anonymous way. For the same reason, we assessed every parent independently; therefore, it was not possible to compare the correlation with an affected child of a single positive parent and both parents' positivity. On the other hand, the guarantee of anonymity provided a parental participation rate of almost $100 \%$, which in turn, represents the strength of this study. A further point of strength was the case-control design, which allowed additional comparative results.

\section{Conclusions}

This study demonstrated a high rate of symptoms consistent with ADHD during childhood in parents of children affected by this pathology. Physicians should actively investigate parents' history when evaluating children with a suspected ADHD syndrome. A proper assessment of the parents of these patients would also be crucial to optimize psycho-educational results.

List of abbreviations: ADHD, Attention Deficit and Hyperactivity Disorder; IQ, Intellective Quotient; WURS25, Wender Utah Rating Scale-25; IQR, Interquartile Range 
DECLARATIONS

Ethics approval and consent to participate: The study was approved by the Institutional Review Board of the Institute for Maternal and Child Health - IRRCCS "Burlo Garofolo", where it was conducted, and all participants gave their written consent to take part in it.

Consent for publication: Patients and/or their caregivers gave written consent for data publication.

Availability of data and materials: All data generated or analysed during this study are included in this published article.

Competing interests: The authors declare that they have no competing interests.

Funding: The authors had no sources of funding to disclose.

Authors' contributions: AS designed the study, GP collected the data; VR performed the statistical analysis; LCW and FR drafted the work, and EB edited the manuscript; all the authors approved the final version of the manuscript and take full responsibility for its contents.

Acknowledgements: The authors thank Martina Bradaschia for the English revision of the manuscript. 


\section{REFERENCES}

${ }^{1}$ American Psychiatric Association (2013). Diagnostic and Statistical Manual of Mental Disorders (DSM-5).

2 Barkley RA, Fischer M, Edelbrock C, Smallish L. The adolescent outcome of hyperactive children diagnosed by research criteria--III. Mother-child interactions, family conflicts and maternal psychopathology. J Child Psychol Psychiatry. 1991 Jan;32(2):233-55.

${ }^{3}$ Kessler RC, Adler LA, Barkley R, Biederman J, Conners CK, Faraone SV, Greenhill LL, Jaeger S, Secnik K, Spencer T, Ustün TB, Zaslavsky AM. Patterns and predictors of attention-deficit/hyperactivity disorder persistence into adulthood: results from the national comorbidity survey replication. Biol Psychiatry. 2005 Jun 1;57(11):1442-51.

${ }^{4}$ Ianes D, Marzocchi GM, Sanna G. (2009). L'iperattività. Aspetti clinici e interventi psicoeducativi, Erickson, Trento.

${ }^{5}$ Biederman J, Mick E, Faraone SV. Age-dependent decline of symptoms of attention deficit hyperactivity disorder: impact of remission definition and symptom type. Am J Psychiatry. 2000 May;157(5):816-8.

${ }^{6}$ Scahill L, Schwab-Stone M, Merikangas KR, Leckman JF, Zhang H, Kasl S. Psychosocial and clinical correlates of ADHD in a community sample of school-age children. J Am Acad Child Adolesc Psychiatry. 1999 Aug;38(8):976-84.

${ }^{7}$ Marzocchi GM, Sclafani M, Rinaldi R, Giangiacomo A. 2012. ADHD in pediatria. Guida operativa, Erickson, Trento.

8 Gizer IR, Ficks C, Waldman ID. Candidate gene studies of ADHD: a meta-analytic review. Hum Genet. 2009 Jul;126(1):51-90.

${ }^{9}$ Burt SA. Rethinking environmental contributions to child and adolescent psychopathology: a meta-analysis of shared environmental influences. Psychol Bull. 2009 Jul;135(4):608-37.

${ }^{10}$ Faraone SV, Perlis RH, Doyle AE, Smoller JW, Goralnick JJ, Holmgren MA, Sklar P. Molecular genetics of attentiondeficit/hyperactivity disorder. Biol Psychiatry. 2005 Jun 1;57(11):1313-23.

${ }^{11}$ Wood AC, Neale MC. Twin studies and their implications for molecular genetic studies: endophenotypes integrate quantitative and molecular genetics in ADHD research. J Am Acad Child Adolesc Psychiatry. 2010 Sep;49(9):874-83.

12 Bidwell LC, Willcutt EG, McQueen MB, DeFries JC, Olson RK, Smith SD, Pennington BF. A family based association study of DRD4, DAT1, and 5HTT and continuous traits of attention-deficit hyperactivity disorder. Behav Genet. 2011 Jan;41(1):165-74.

${ }^{13}$ Ward MF, Wender PH, Reimherr FW. The Wender Utah Rating Scale: an aid in the retrospective diagnosis of childhood attention deficit hyperactivity disorder. Am J Psychiatry. 1993 Jun;150(6):885-90. 
${ }^{14}$ McCann BS, Scheele L, Ward N, Roy-Byrne P. Discriminant validity of the Wender Utah Rating Scale for attentiondeficit/hyperactivity disorder in adults. J Neuropsychiatry Clin Neurosci. 2000 Spring;12(2):240-5.

15 National Collaborating Centre for Mental Health. Attention deficit hyperactivity disorder: diagnosis and management of ADHD in children, young people, and adults. Clinical Guideline 27. London: National Institute for Health and Clinical Excellence; 2008.

${ }^{16}$ Weiss M, Hechtman L, Weiss G. ADHD in parents. J Am Acad Child Adolesc Psychiatry. 2000;39(8):1059-1061.

17 Sonuga-Barke EJS, Daley D, Thompson M. Does maternal ADHD reduce the effectiveness of parent training for preschool children's ADHD? J Am Acad Child Adolesc Psychiatry. 2002;41(6):696-702.

18 Starck M, Grünwald J, Schlarb AA. Occurrence of ADHD in parents of ADHD children in a clinical sample. Neuropsychiatr Dis Treat. 2016 Mar 3;12:581-8.

${ }^{19}$ Friedman LM, Rapport MD, Raiker JS, Orban SA, Eckrich SJ. Reading Comprehension in Boys with ADHD: The Mediating Roles of Working Memory and Orthographic Conversion. J Abnorm Child Psychol. 2017 Feb;45(2):273-287.

${ }^{20}$ Iglesias-Sarmiento V, Deaño M, Alfonso S, Conde Á. Mathematical learning disabilities and attention deficit and/or hyperactivity disorder: A study of the cognitive processes involved in arithmetic problem solving. Res Dev Disabil. 2017 Feb;61:44-54.

${ }^{21}$ Tabassam W, Grainger J (2002). Self-concept, attributional Style and Self-efficacy Belief of Student with learning disabilities with and without Attention Deficit Hyperactivity Disorder, in "Learning Disability Quarterly", 25 n.2, 14151.

22 Hinshaw SP, Carte ET, Sami N, Treuting JJ, Zupan BA. Preadolescent girls with attention-deficit/hyperactivity disorder: II. Neuropsychological performance in relation to subtypes and individual classification. J Consult Clin Psychol. 2002 Oct;70(5):1099-111. 


\section{TABLES}

Table 1. Parents stratified by study group and WURS-25 test result (positive/negative).

\begin{tabular}{|c|c|c|c|c|c|c|c|}
\hline & \multicolumn{3}{|c|}{ Parents of ADHD children } & \multicolumn{3}{|c|}{ Parents of non-ADHD children } & \multirow[b]{2}{*}{ p-value } \\
\hline & N. & $\begin{array}{c}\text { Negative } \\
\text { test }\end{array}$ & Positive test & N. & Negative test & Positive test & \\
\hline Total & 117 & $71(60.7 \%)$ & $46(39.3 \%)$ & $\begin{array}{c}12 \\
0\end{array}$ & $118(98.3 \%)$ & $2(1.7 \%)$ & $<0.0001$ \\
\hline Mothers & 60 & $42(70 \%)$ & $18(30 \%)$ & 60 & $59(98.3 \%)$ & $1(1.7 \%)$ & $<0.0001$ \\
\hline Fathers & 57 & $29(50.9 \%)$ & $28(49.1 \%)$ & 60 & $59(98.3 \%)$ & $1(1.7 \%)$ & $<0.0001$ \\
\hline
\end{tabular}

Table 2. Median and interquartile range (in parenthesis) score of questions assessing school difficulties and emotional functioning stratified by sex with Wilcoxon-Mann-Whitney test $p$-values.

\begin{tabular}{|c|c|c|c|c|c|c|}
\hline & & \multicolumn{2}{|c|}{ Positive } & \multicolumn{2}{|c|}{ Negative } & \multirow[b]{2}{*}{$p$-value } \\
\hline & & N. & Median & N. & Median & \\
\hline \multirow{3}{*}{ School difficulties } & Mothers & 19 & $9.0(9)$ & 101 & $3.0(8)$ & $<0.0001$ \\
\hline & Fathers & 29 & 8.00 (9) & 88 & $3.00(9)$ & $<0.0001$ \\
\hline & Total & 48 & $8.0(9)$ & 189 & $3.0(9)$ & $<0.0001$ \\
\hline \multirow{3}{*}{ Emotional deregulation } & Mothers & 19 & $14.0(5)$ & 101 & $5.0(14)$ & $<0.0001$ \\
\hline & Fathers & 29 & $13.0(8)$ & 88 & $5.0(15)$ & $<0.0001$ \\
\hline & Total & 48 & $14.0(8)$ & 189 & $5.0(15)$ & $<0.0001$ \\
\hline
\end{tabular}


FIGURE LEGEND

Figure 1. Co-morbidities in non-ADHD patients

Figure 2. The Wender Utah Rating Scale (WURS) 25

Figure 3. Scores distribution of the WURS-25 test comparing cases and controls, and on questions investigating school impairment and emotional deregulation comparing subjects with an overall positive or negative test. 


\section{Figures}

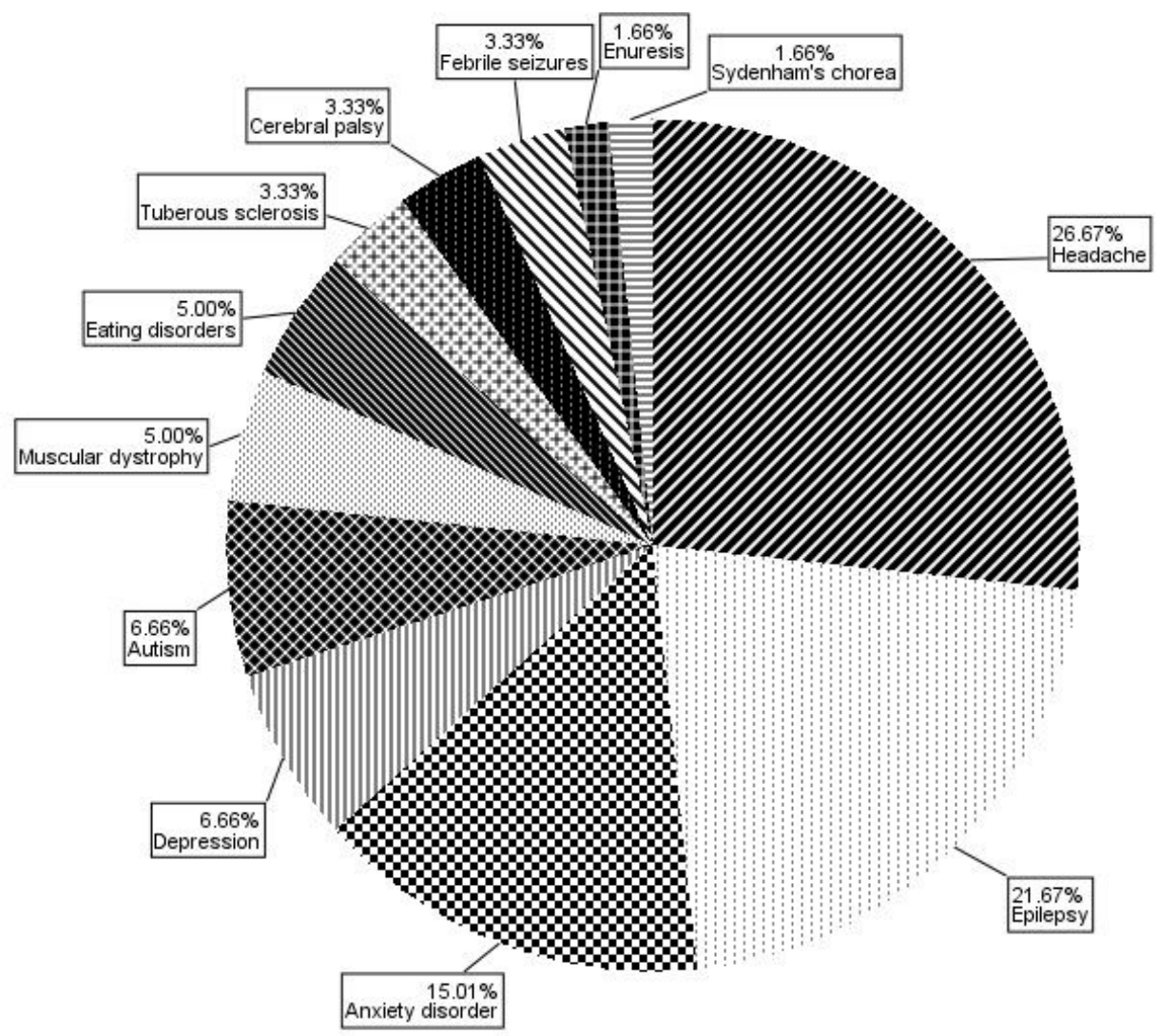

Z. Headache Epilepsy

WAnxiety disorder

IIII Depression

Autism

Muscular dystrophy

$\mathbb{N}$ Eating disorders

it Tuberous sclerosis

Cerebral palsy

NFebrile seizures

HEnuresis

三Sydenham's chorea

\section{Figure 1}

Co-morbidities in non-ADHD patients 
- As a child I was (or had):

\begin{tabular}{|c|c|c|c|c|c|c|}
\hline & & 0 & 1 & 2 & 3 & 4 \\
\hline 1 & Easily distracted & & & & & \\
\hline 2 & Anxious worrying & & & & & \\
\hline 3 & Nervous fidgety & & & & & \\
\hline 4 & Inattentive daydreaming & & & & & \\
\hline 5 & Hot- or short-tempered & & & & & \\
\hline 6 & Temper outbursts tantrums & & & & & \\
\hline 7 & Trouble not finishing things & & & & & \\
\hline 8 & Stubborn strong-willed & & & & & \\
\hline 9 & Sad or blue depressed & & & & & \\
\hline 10 & Disobedient with parents & & & & & \\
\hline 11 & Low opinion of myself & & & & & \\
\hline 12 & Irritable & & & & & \\
\hline 13 & Moody ups and downs & & & & & \\
\hline 14 & Angry & & & & & \\
\hline 15 & Impulsive, acting without thinking & & & & & \\
\hline 16 & Tendency to be immature & & & & & \\
\hline 17 & Guilty feelings regretful & & & & & \\
\hline 18 & Losing control of myself & & & & & \\
\hline 19 & Tendency to be or act irrationally & & & & & \\
\hline 20 & Unpopular, not very friendly & & & & & \\
\hline 21 & Trouble seeing someone else's view & & & & & \\
\hline 22 & Trouble with authorities & & & & & \\
\hline 23 & Overall a poor student & & & & & \\
\hline 24 & Troubles with math or numbers & & & & & \\
\hline 25 & Not achieving up to potential & & & & & \\
\hline
\end{tabular}

Where: 0 = Not at all or slightly; 1 = Mildly; 2 = Moderately; 3 = Quite a bit; 4 = Very much

- Has you repeated classes? (indicate which)

Figure 2

The Wender Utah Rating Scale (WURS) 25 

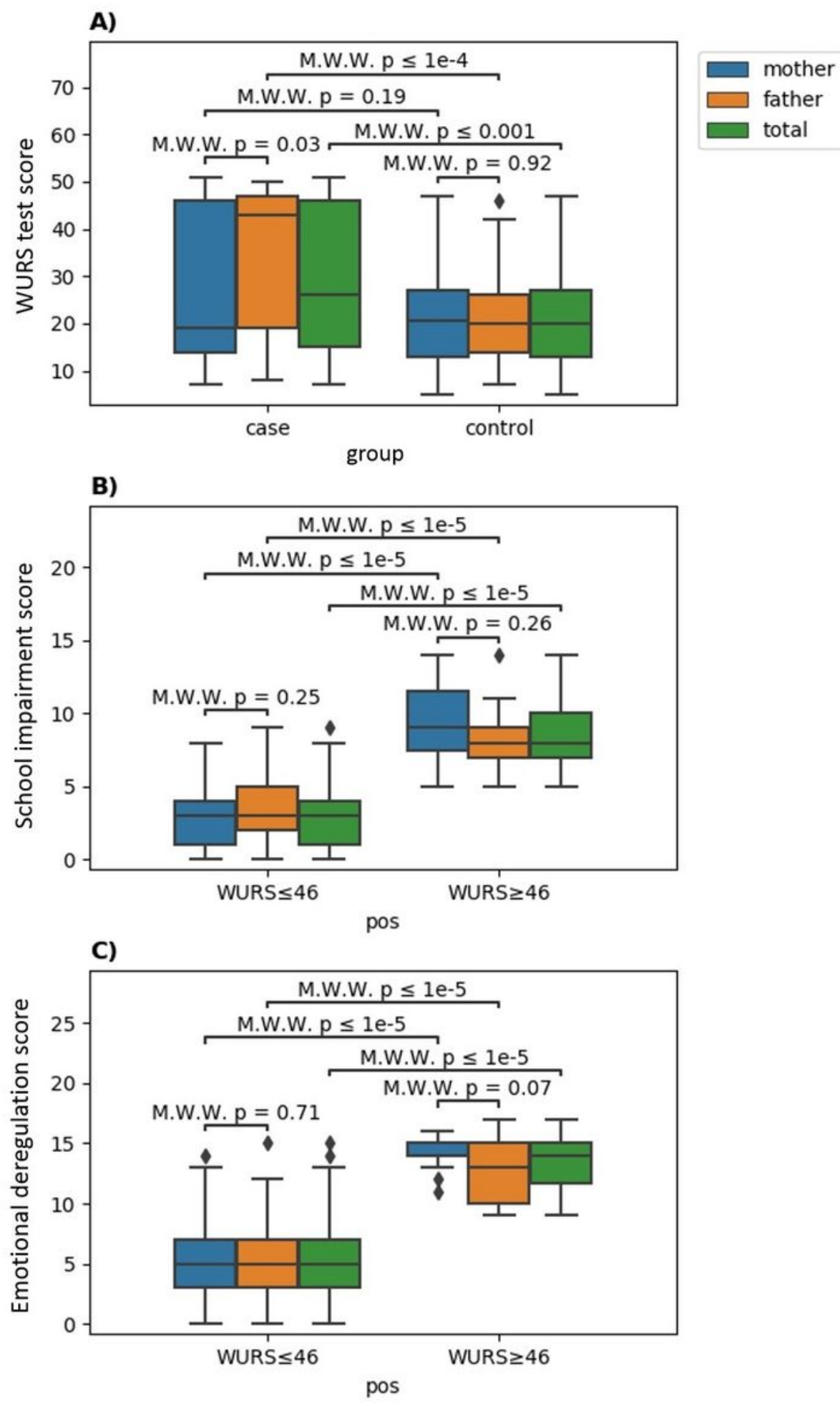

\section{Figure 3}

Scores distribution of the WURS-25 test comparing cases and controls, and on questions investigating school impairment and emotional deregulation comparing subjects with an overall positive or negative test. 